\title{
Transparent Membrane of Polyelectrolyte Complex Doped with 2,6-Dichlorophenol-Indophenol as an Optical Sensor for Colorimetric Measurements of Ascorbic acid
}

\section{DHONY HERMANTO ${ }^{1,2 *}$, NURUL ISMILLAYLI ${ }^{1}$, RURU HONIAR ${ }^{2}$, I. G. AYU SRI ANDAYANI ${ }^{2}$, BAIQ MARIANA ${ }^{2}$ and ROCHMAD KRIS SANJAYA ${ }^{3}$}

\author{
${ }^{1}$ Department of Chemistry, Faculty of Mathematics and Natural Sciences, University of Mataram, \\ Mataram-West Nusa Tenggara 83125, Indonesia. \\ ${ }^{2}$ Analytical Chemistry Laboratory, Faculty of Mathematics and Natural Sciences, University of Mataram, \\ Mataram-West Nusa Tenggara 83125, Indonesia. \\ ${ }^{3}$ Department of Pharmacy, Faculty of Healthy Science, University of Kadiri, \\ Kediri-East Java 64115, Indonesia. \\ ${ }^{*}$ Corresponding author E-mail: dhony.hermanto@ unram.ac.id \\ http://dx.doi.org/10.13005/ojc/360412
}

(Received: July 15, 2020; Accepted: August 16, 2020)

\begin{abstract}
An optical chemical sensor based on a transparent membrane of polyelectrolyte complex (PEC), composed of alginate-chitosan membrane and 2,6-dichlorophenol-indophenol (2,6-DCPIP), coated on transparent mica was coupled with UV-Vis spectrophotometry and used for colorimetric measurements of ascorbic acid (AA). The transparent membranes of PEC were characterized using scanning electron microscopy (SEM) technique. The experimental parameters of the biosensor were optimized. Here, the optical chemical sensor showed maximum response at wavelength of $545 \mathrm{~nm}$, with the optimum $\mathrm{pH}$ at 3 . The calibration curve had a dynamic working range at 0.0 to $5.0 \mathrm{mM}$ of AA with a limit of detection (LOD) of $0.13 \mathrm{mM}$ AA. The membrane sensor has a long life time in the defined condition $\left(4^{\circ} \mathrm{C}\right)$. The developed optical chemical sensor correlated well with the standard method, HPLC for the determination of Ascorbic acid concentration in real samples.
\end{abstract}

Keywords: Alginate-chitosan PEC membrane, DCPIP, Colorimetric sensor, Ascorbic acid.

\section{INTRODUCTION}

In recent years, the development of optical sensors as analyte detection device in environmental, chemistry and biotechnology science, as well as in the pharmaceutical and health industry, have received great attention from researchers ${ }^{1}$. Colorimetric sensors as a type of optical chemical sensor is very interesting due to its ability in detecting analytes through a change in color ${ }^{2}$. This feature makes it possible to obtain visual and analytical signal readings which is easily scalable. This chemical sensor usually contains chromogenic materials that are immobilized in the supporting matrix $^{3}$. Many parameters such us mechanical properties, stability, suitability and permeability of

This is an Open Access article licensed under a Creative Commons license: Attribution 4.0 International (CC- BY). Published by Oriental Scientific Publishing Company @ 2018

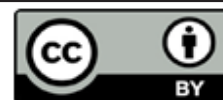


chromogenic material influence the selection of appropriate supporting matrix. Polymers with many desirable features are widely used as supporting materials for various optical chemical sensors ${ }^{4}$. Currently, polymers such us polyvinyl chloride (PVC), polystyrene (PS), polytetrafluoroethylenes (PTFE), polydimethylsiloxanes (PDMS), polymethacrylate (PMA) and their derivative cellulose such as ethyl cellulose have been used widely ${ }^{5}$.

In the last decade, PEC has drawn attention from various scientific disciplines to be applied as a supporting matrix. PEC is an association complex formed between polymers that have polyions with opposite charges trough electrostatic interaction ${ }^{6}$. Alginate which is polyanionic and polycationic chitosan when dissolved in the right conditions can interact each other through carboxyl groups from alginate and amino groups from chitosan ${ }^{7}$. The formed PEC is expected to provide better applications due to its unique structure and properties ${ }^{8}$. Recently, it has been developed a sensor using PEC alginate-chitosan matrix as immobilized membrane of bioactive material ${ }^{9}$. The membrane is suitable for immobilizing active material to produce a selective sensing interface for detection of target analytes ${ }^{10}$. In this case, a fast and very sensitive response is expected because of the large specific surface area, and rapid penetration of the sample solution into the pores of the membrane. These features facilitate the rapid interaction of target analytes with a large number of active sites that can be accessed on the membrane network. The use of natural alginate-chitosan membrane in optical chemical sensors with spectroscopy requires a transparent membrane.

In this research optical chemical sensor fabrication based on alginate-chitosan doped with 2,6-DCPIP transparent membranes was carried out, then sensors were applied for colorimetric detection of AA using a spectrophotometer. The role of vitamin $C$ in metabolic function is very important, especially in development and maintenance of connective tissue, activation vitamin B and folic acid and conversion of cholesterol to bile acid and tryptophan to serotonin ${ }^{11}$. As antioxidant, vitamin C protects the body from radical damage as well as immune system by reducing the effect of allergies and fighting infection ${ }^{12,13}$.
The developed optical sensor was built by forming a transparent $\mathrm{PEC}$ membrane from alginatechitosan and 2,6-DCPIP membrane coated on transparent mica. For the detection of AA colorimetry, the sample solution was contacted with an optical sensor. The colorimetric reaction is based on the 2,6DCPIP interaction as an indicator with Ascorbic acid ${ }^{14}$. This is the first time that optical sensor colorimetry based on transparent PEC membranes has been made and coupled with UV-Vis spectrophotometry for colorimetric detection of ascorbic acid.

\section{EXPERIMENTAL}

\section{Materials and Instruments}

Chemicals consisting of 2,6-dichlorophenolindophenol, sodium alginate of brown algae (300 to $400 \mathrm{cp}$ ), and chitosan of crab shells (95\% deacetylation) were purchased from Sigma (St. Lois, USA). Hydrochloric acid (37\%), glacial acetic acid (98\%), metaphosphoric acid, sodium hydroxide, sodium bicarbonate and ascorbic acid were received from Merck \& Co. (UK). A standard solution of ascorbic acid (only prepared at the time of use) was prepared by dissolving $50 \mathrm{mg}$ of ascorbic acid with solution of metaphosphate-acetic acid into a $50 \mathrm{~mL}$ volumetric flask. The methosphoric acid solution was prepared by mixing $100 \mathrm{~mL}$ of deionized distilled water (dd) with $20 \mathrm{~mL}$ of acetic acid, and $7.5 \mathrm{~g}$ of metaphosphoric acid. The mixture was diluted up to $250 \mathrm{~mL}$ with dd water under stirring condition. The indophenol coloring solution was prepared by mixing $50 \mathrm{~mL}$ of water, $42 \mathrm{mg}$ of sodium bicarbonate, and $50 \mathrm{mg}$ of 2,6-dichloroindophenol sodium salt. The mixture was diluted to $200 \mathrm{~mL}$ with dd water and stirred until homogeneous. Each metaphosphoric acid-acetic acid solution and indophenol dye solution were filtered and then stored at a closed container in the refrigerator until use.

\section{Preparation of Transparent PEC Membrane}

The membrane was prepared from hydrosols of chitosan and alginate, as described by Hermanto et al.6,9. The scanning electron microscope (SEM) study was carried out on a transparent PEC membrane after being coated with gold palladium on SEM (Joel LV 5600 USA model) to evaluate the morphology and structure of membrane surface, as shown in Fig. 1. Here, the morphology of the alginatechitosan membrane mixed was less homogeneous, it can be observed that the transparent membrane 
surface of the alginate-chitosan shows a fibrous structure with a rough morphology and irregular shape, which is a network of electrostatic interactions of alginate and chitosan. The likeness of its surface morphology has been described at previously work $^{6,9}$.

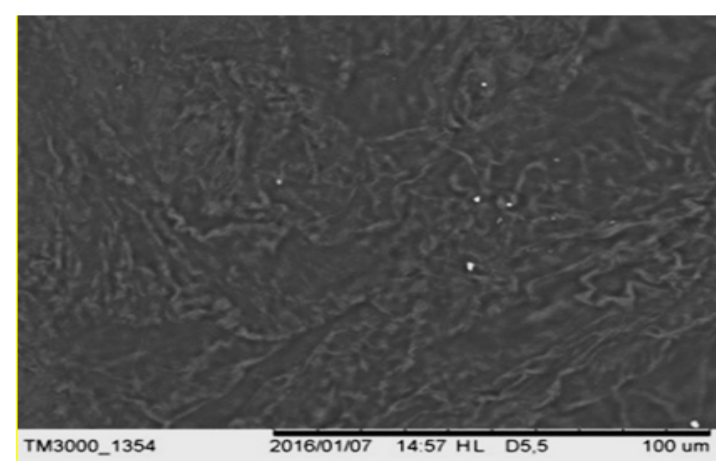

Fig. 1. SEM images of surface morphology of transparent membrane of alginate-chitosan

\section{Immobilization Procedure}

Alginate-chitosan hydrosol mixture was used as a supporting matrix for 2,6-DCPIP (as an indicator of the colorimetric optical system). Before the hydrosol mixture (50 $\mathrm{mL}$ alginate- chitosan) was used, the mixture was added with $2 \mathrm{~mL}$ of $32 \%(\mathrm{v} / \mathrm{v})$ $\mathrm{HCl}$ and $10 \% \mathrm{NaOH}(\mathrm{w} / \mathrm{v})$ to reach approximately $\mathrm{pH}$ of 5.28. Stirring was carried out for $\pm 4 \mathrm{~h}$ at room temperature until the mixture was homogeneous. After that, $3 \mu \mathrm{L}$ hydrosol mixture was taken and added to $1 \mathrm{~L}$ phosphate buffer $(\mathrm{pH} 6)$. Then, $3 \mu \mathrm{L}$ indophenol dye solution was added to the buffered mixture. Immediately, the transparent mica coated with alginate-chitosan hydrosol mixture were used for indophenol immobilization. The mixture was stored for 5 days in the refrigerator until the membrane was formed. The final membrane was removed from the mold and stored in a closed container and was kept in the refrigerator for the aging process until use.

\section{Measurement Procedure}

Alginate-chitosan/indophenol membrane was added to AA in a buffer phophate solution $(\mathrm{pH}$ 6.0) at a specified time (5 s). The membrane was rinsed with water and then dried. Film absorbance was measured using spectrophotometer at maximum wavelength. This procedure allows measurement of absorbance in a colored or turbid sample, such as an orange/apple juice sample. To evaluate the feasibility of the optical chemical sensor for colorimetric measurements of $A A$ detection in the real sample, the sample orange/apple juice was first filtered with a $0.22 \mu \mathrm{m}$ syringe filter and then spiked with standard AA solutions to prepare stock solutions with various $A A$ concentrations. The optical intensity was recorded on UV-Vis spectrophotometer under the same conditions. As a comparison, the HPLC was used as the reference method for the determination of $A A$ in the real sample, recommended by AOAC official method 2012.2215. The HPLC used is a product from Waters Breeze HPLC-USA (which is equipped with various devices, namely Waters Delta-600 pump, Waters 600-controller, Waters-2487 Dual I Absorbance Detector and column SGE type 250CL4-ODS2-8/5 $\mu \mathrm{m}$, length $250 \mathrm{~mm}, 4 \mathrm{~mm}$ dan frit $4 / 5 \mu \mathrm{m})$. Real samples were analyzed by HPLC using a standard internal method with a UV detector at a maximum wavelength of $245 \mathrm{~nm}$. A calibration curves for $A A$ in the concentration range of 0.0 and $10 \%(\mathrm{v} / \mathrm{v})$ was obtained using $96 \%$ ethanol as a solvent. The sample was previously diluted to obtain an adequate AA concentration.

\section{RESULTS AND DISCUSSION}

\section{Sensor Scheme}

Ascorbic acid concentration in this study was determined by optical chemical sensors for colorimetric measurements based on a transparent membrane of PEC, composed of alginate-chitosan membrane and 2,6-DCPIP, coated on transparent mica was coupled with UV-Vis spectrophotometry. The principle for determining AA concentration is the presence of AA oxidation by 2,6-DCPIP which is a color indicator to dehydroascorbic acid ${ }^{16}$. AA will donate one electron to form semidehydroascorbic acid which then undergoes disproportionation to form dehydroascorbic acid which is unstable. Dehydroascorbic acid would be degraded to form oxalic acid and treonic acid. At the same time, the 2,6-DCPIP indicator becomes a colorless material (leucodye). The 2,6-DCPIP solution is blue in neutral or basic conditions and rose pink in acidic conditions ${ }^{14}$. Redox reactions between indicators (DCPIP) and analytes (AA) can be represented as follows:

$$
\underset{\text { rosepink }}{\mathrm{DCPIP}} \text { ox }_{\text {colorless }}+\underset{\text { colorless }}{\mathrm{A} A_{\text {red }}} \rightarrow \underset{\text { colorless }}{\mathrm{DCPIP}}
$$

Where 'ox' and 'red' refer to oxidized and reduced forms of each species. Here, there are two half reactions: DCPIP $_{\text {ox }}$ accepted electrons to be DCPIP red and AAred became $\mathrm{AA}_{\text {ox }}$ by releasing 
electrons, where electron transfer occured between the two species. Once the AA analyte reacted with the DCPIP indicator, the AA was oxidized and the DCPIP indicator was reduced, i.e. there was a loss of electrons in the former process and a gain of electrons in the latter process. The DCPIP indicator changes from a color oxidized form to a colorless reduced form. Therefore, the absorbance intensity of the DCPIP indicator decreased with decreasing of indicator due to the interaction between $A A$ and DCPIP. Hence, this can be used as a basic principle for the measurement of $A A$ in solution. This principle has also been observed in AA titrimetry analysis using 2,6-DCPIP ${ }^{17,18}$.

\section{Optimization of the Colorimetric Measurements} Optimization of experimental parameters is an important step in the analytical characteristics of optical chemical sensors performance for the colorimetric measurement of AA. To determine the optimal operating parameters, a series of initial investigations were carried out and Table 1 showed the range of each parameter and its optimal value. Parameters can be grouped into physical and chemical dimensions.

Table 1: Experimental parameters of optical sensor performance for AA measurement

\begin{tabular}{lccc}
\hline Dimensions & Parameter & Range & Optimum Value \\
& Wave length $\lambda_{\max }(\mathrm{nm})$ & $400-800$ & 545 \\
Physical & Respon time $(\mathrm{s})$ & $0-60$ & 5 \\
& Temperature $\left({ }^{\circ} \mathrm{C}\right)$ & $20-35$ & 25 \\
Chemical & $\mathrm{pH}$ & $2-6$ & 3 \\
& Volume of indicator & $0.1-0.5$ & 0.2 \\
\hline
\end{tabular}

One important aspect in physical/chemical parameters to determine the optimal conditions of optical chemical sensors for colorimetric $A A$ measurements is scanning wavelength, response time and temperature. The optimum wavelength as the best operational wavelength of an optical chemical sensor, was determined by measuring the difference in signal intensity before and after reacting with the analyte $(A A)$. This change in signal intensity occured due to color change of dye when a redox reaction occurs with the interaction between the analyte and the dye. The intensity of the absorbance signal decreased because the indicator color changes from pink-rose to colorless. Monochromator was used to monitor changes in signal intensity at wavelength of $545 \mathrm{~nm}$, where the absorbance difference of reduced and oxidized forms was the greatest. In addition, the sensor response time was the needed time for an optical chemical sensor reaching a stable signal intensity. The sensor response time was measured in range from 0.0 to $20 \mathrm{~s}$. It was found that 5 seconds is the response time of the optical chemical sensor, because after $5 \mathrm{~s}$, the sensor signal did not increase significantly with increasing time. The working temperature of the redox reaction affects changes in blank and redox signals. It was obtained that the room temperature, $25^{\circ} \mathrm{C}$ was the optimum temperature for this redox reaction. At lower temperatures redox activity decreased due to the limited movement of the analyte into the pore membrane (less elastic membrane) although AA analytes can be protected from the effects of oxidation by the environment at low temperatures. At higher temperatures, redox activity decreased because AA molecules are easily oxidized at relatively high temperatures. The temperature of $25^{\circ} \mathrm{C}$ was then chosen as the working temperature on the optical chemical sensor for the measurement of colorimetric Ascorbic acid.

In chemical dimensions, the parameters directly involved in the redox process, namely $\mathrm{pH}$ and the amount of dye solution. The redox reaction indicator 2,6-DCPIP followed the change from oxidized to reduced form and involved the transfer of electrons and $\mathrm{H}^{+}$ions in their reactions so that the reaction depends on $\mathrm{pH}$. The effect of $\mathrm{pH}$ ws examined by comparing the sensor characteristic response at $\mathrm{pH}$ 3.0-6.0. The linear range of sensors observed shifted to lower concentrations when the $\mathrm{pH}$ increased. At $\mathrm{pH} 6.0$ the sensor response was observed to be lower than the value at $\mathrm{pH}$ 3.0. This is due to a decrease in the formal potential of the indicator as a result of an increase in $\mathrm{pH}$. The formal potential of 2,6-DCPIP has previously been observed to decrease with increasing $\mathrm{pH}$. A decrease in formal potential indicates that the indicator was more easily reduced, thus lower analytic concentrations could cause the indicator changed color. This also implies that the $\mathrm{pH}$ of the analyte solution during measurements must be kept as constant as possible. In this study, phosphate buffer solution at $\mathrm{pH} 3$ was found to be optimum for use in the redox reaction. The buffer solution was used to minimize the influence of the matrix and other electrocatalytic interference so that the stability of the sensor response to changes in $\mathrm{pH}$ can be controlled. In addition, concentration of 
immobilized indicator in the matrix was determined at optimum conditions for obtaining a high and constant signal intensity. Measurement of dye solution volume with variations $0.1,0.2,0.3,0.4$ and $0.5 \mathrm{~mL}$ was conducted and the optimum volume was $0.2 \mathrm{~mL}$.

\section{Response optical sensor for colorimetric measurements of Ascorbic acid}

The response of optical sensors for colorimetric measurements of AA has been investigated under optimal conditions. The optical sensor response curve for colorimetric measurements of AA showed change in optical intensity due to change in AA concentration. Fig. 2 is an optical sensor calibration curve for colorimetric measurements of AA at wavelength of $545 \mathrm{~nm}$.

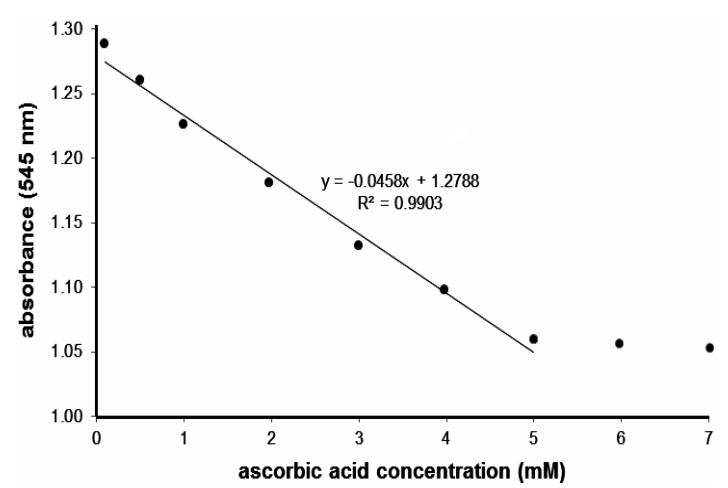

Fig. 2. The calibration plot of AA measurement

Figure 2 show a calibration curve plot between optical signal intensity and AA concentration. The curve linearity is good with the correlation coefficient, $\left.R^{2}=+0.9903(r) 1\right)$ in the range of $A A$ ion concentrations between 0.0 to $5.0 \mathrm{mM}$. The negative value of the gradient showed a redox decrease in proportion to its concentration. To calculate limit of detection for colorimetric measurements of $\mathrm{AA}$, the membrane was immersed in a phosphate buffer solution $(\mathrm{pH}=3$ ) without AA (blank solution) for $5 \mathrm{~s}$ and then the absorbance was measured at $545 \mathrm{~nm}$. Limit of detection of biosensor is $0.13 \mathrm{mM}$.

Reproducibility and repeatability of optical chemical sensors were two important characteristics studied in this work. Reproducibility and repeatability are the ability of the biosensor to provide the same output when given a fixed input after the system is reset. Repeatability of experiments is expected to produce the difference in results between experiments as small as possible or produce precise data. Reproducibility of the optical sensor for colorimetric measurements of AA at a wavelength of $545 \mathrm{~nm}$ was determined by making three measurements using three different membranes. The results showed that at least 99\% reproducibility and membranes made on different days showed no statistically significant differences $(\alpha=0.05)$. Meanwhile, optical chemical sensor repeatability was determined as the relative standard deviation (RSD) in determining the standard $A A$ solution $(n=3, A A 1=$ first membrane to $A A 3=$ third membrane) using the same membrane in the concentration range of 0.0 to $7.0 \mathrm{mM}$. Its value was 0.0 up to $0.98 \%$. This RSD are higher when compared with other reported for determination AA-based optical sensor ${ }^{19,20}$. This show that optical chemical sensors have excellent reproducibility and repeatability, as shown in Figure 3.

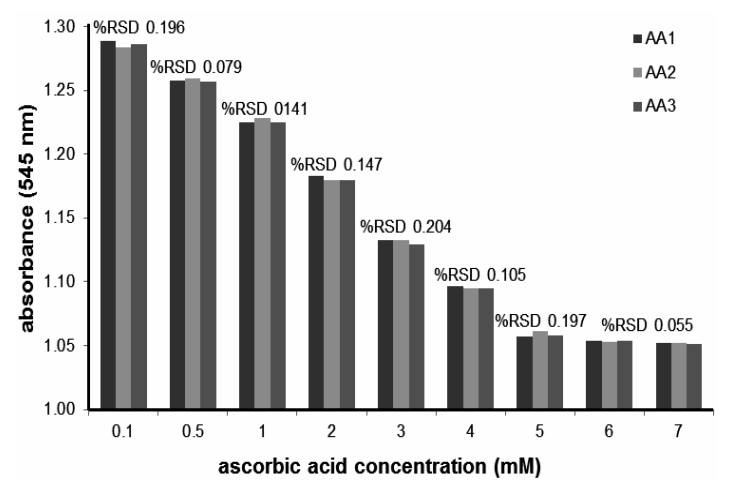

Fig. 3. The optical sensor for colorimetric response toward reproducibility at various concentration of AA (at absorbance $545 \mathrm{~nm}$ and $0.5 ; 0.1 ; 1 ; 2 ; 3 ; 4 ; 5 ; 6$ and $7 \mathrm{mM}$ AA standard solution, and $\mathrm{n}=3$ )

Analytical characteristics of this method for the determination of Ascorbic acid

Optical chemistry sensor based on transparent doped membrane with 2,6-DCPIP has the advantage that can be reused through reactivation by flushing using $1 \mathrm{mM}$ of $\mathrm{K}_{3} \mathrm{Fe}(\mathrm{CN})_{6}$ solution. The lifetime or reuse of optical chemical sensor was determined from the decreased redox activity due to the leaching effect of the dye from the membrane cavity at a certain time. Besides being caused by redox reaction with $A A$, activity of immobilized indicator was also reduced during storage due to interacting with air and high temperatures. To minimize this factor, immobilized dyes were stored in the refrigerator when not in used. Fig. 3 shows the use of optical chemical sensors for the measurement of colorimetric AA-based transparent membrane alginate-chitosan doped with 2,6-DCPIP for 10 times the use after reactivation. Absorbance readings on the first day were considered as $100 \%$ relative response. As shown in Fig. 4, the optical chemical sensor response was stable for period of 18 days with maintaining more than $75 \%$ of the 
initial response. However, the response of the optical chemical sensor decreased dramatically to $45 \%$ of the initial response during the 20 days of its storage. It indicated that the optical chemical sensor was stable for 10 times usage.

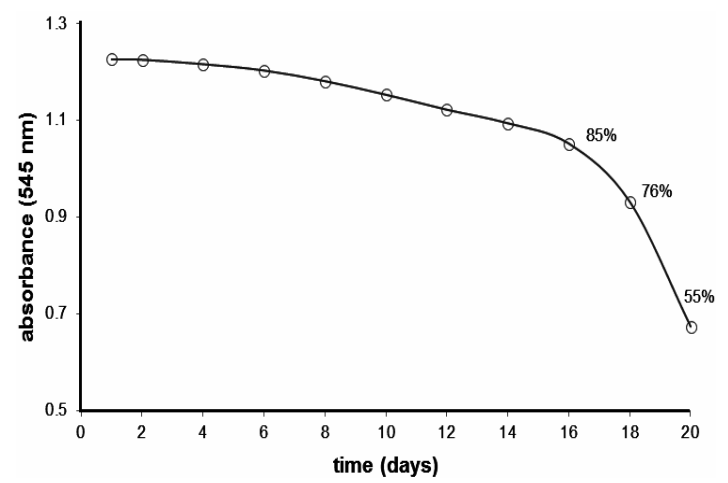

Fig. 4. Stability of optical chemical sensor

The selectivity of optical chemical sensors was determined by adding interference that was potentially disturbing and commonly found in real samples such as glucose, caffeine, boric acid, lactic acid and citric acid. Table 2 shows the activity of optical chemical sensors for the measurement of colorimetric AA at several interferences. The analysis was carried out with the ratio of $A A$ concentration to the interferent of 1:10 (1 mM AA, $10 \mathrm{mM}$ interference). Redox values were relatively very small $(<5 \%)$, indicating that the interferent does not have a significant effect. However, citric acid and boric acid had relatively greater values compared to other interferences because citric acid and boric acid at high concentrations could affect redox activity at 2,6-DCPIP.

Table 2: Determination results of possible interferences tested with the optical sensor

\begin{tabular}{lcc}
\hline Interference & Relative Redox Activity (\%) & Recovery (\%) \\
\hline Glucose & $+0.55 \pm 0.05$ & 105.6 \\
Cafein & $+0.46 \pm 0.06$ & 103.1 \\
Boric Acid & $+1.78 \pm 0.13$ & 99.2 \\
Lactic Acid & $+1.55 \pm 0.11$ & 99.0 \\
Citric Acid & $+1,86 \pm 0.14$ & 99.4 \\
\hline
\end{tabular}

Notes: Percentage of interfering or percentage of relative redox activity (\%): $((a-b) / b) \times 100 \%$; a: absorbance for the mixture of interfering compound and AA; b: absorbance for AA (without interfering compound)

\section{Detection of AA in a Real Sample}

The performance of optical chemical sensors were evaluated in the analysis of real samples. The standard addition method was used by spiking different amounts of AA into the sample. The results of determination are summarized in Table 3. The results indicated that the optical chemical sensor developed has very good characteristics for colorimetric determination of $A A$ in real sample. The results shown in Table 3 were in accordance with the reference method using HPLC.

Table 3: Determination of AA in real samples using purposed optical sensors and HPLC

\begin{tabular}{|c|c|c|c|}
\hline \multirow[t]{2}{*}{ Sample } & \multicolumn{2}{|c|}{ AA concentration (mg in $100 \mathrm{~g}$ sample) } & \multirow{2}{*}{$\begin{array}{l}\text { Relative } \\
\text { Error (\%) }\end{array}$} \\
\hline & Found by Sensor & Found by HPLC & \\
\hline Orange Juice & $52.66 \pm 0.87$ & $52.91 \pm 0.17$ & 2.9 \\
\hline Apple Juice & $4.13 \pm 0.97$ & $3.82 \pm 0.59$ & 2.5 \\
\hline
\end{tabular}

Notes: average triplicate of measurements.

\section{CONCLUSION}

The 2,6-DCPIP dye was immobilized into the transparent alginate-chitosan membrane and then coated on transparent mica as an optical chemical sensor for colorimetric measurements of ascorbic acid (AA). After optimization of experimental parameters, optical chemical sensors have a linear response in the $A A$ concentration range of 0.0 to $5.0 \mathrm{mM}$ and a detection limit of $0.13 \mathrm{mM}$. Reproducibility and repeatability was excellent (RSD $0.2 \%$ ) as well as its stability up to 10 times usage. Hence, optical chemical sensors based on 2,6-DCPIP/ alginate-chitosan transparent membranes became an alternative method for determining AA levels in samples.

\section{ACKNOWLEDGMENT}

This research did not receive any specific grant from funding agencies in the public, commercial, or not-for-profit sectors.

\section{Conflicts of Interest}

The authors declare no conflict of interest.

\section{REFERENCES}

1. Dey, D.; Goswami, T. J. Biomed. Biotechnol., 2011, 2011, 1-7.

2. Li, Z.; Askim, J. R.; Suslick, K. S. Chem. Rev., 2018, 19(1), 1-62.
3. Wang, X.; Wolfbeis, O. S. Chem. Soc. Rev., 2014, 43, 3666-3761.

4. Gruber, P.; Marques, M. P. C.; Szita, N.; Mayr, T. L. a Chip., 2017, 17, 2693-2712. 
5. Cichosz, S.; Masek, A.; Zaborski, M. Polym. Test., 2018, 67, 342-348.

6. Hermanto, D.; Mudasir, M.; Siswanta, D.; Kuswandi, B.; Ismillayli, N. Molekul., 2020, 15(1), 40-47.

7. Ismillayli, N.; Hadi, S.; Dharmayani, N K. T.; Sanjaya, R. K.; Hermanto, D. IOP Conference Series: Mat. Sci. Eng., 2020, 833, 1-6.

8. Yabuki, S. Anal. Sci. 2011, 27, 695-702.

9. Hermanto, D.; Mudasir, M.; Siswanta, D.; Kuswandi, B.; Ismillayli, N. J. Math. Fundam. Sci., 2019, 51(3), 309-319.

10. Hermanto, D.; Kuswandi, B.; Siswanta, D.; Mudasir, M. Indones. J. Chem., 2019, 19(3), 786-795.

11. Chambial, S.; Dwivedi, S.; Shukla, K. K.; John, P. J.; Sharma, P. Indian J. Clin. Biochem., 2013, 28(4), 314-328.

12. Padayatty, S. J.; Katz, A.; Wang, Y.; Eck, P.;
Kwon, O.; Lee, H.; Chen, S.; Corpe, C.; Dutta, A.; Dutta, S. K. J. Am. Coll. Nutr., 2003, 22(1), 18-35.

13. Carr, A. C.; Maggini, S. Nutrients., 2017, 9(121), 1-25.

14. Elbehery, N. H. A.; Amr, A. E. E.; Kamel, A. H.; Elsayed, E. A.; Hassan, S. S. M. Sensors., 2019, 19(2058), 1-13.

15. AOAC. AOAC Official Method., 2012.22. 2012, 1, 1-2.

16. Sripakdee, T.; Mahachai, R.; Chanthai, S. Orient. J. Chem., 2017, 33(1), 74-86.

17. AOAC. AOAC Official Method., 967.21. 2006, 1, 6-7.

18. Ugbe, F. A.; Ikudayisi, V. A.; Amusan, O. T. Int. Ann. Sci., 2017, 3(1), 19-22.

19. Bsalan, G. A.; Rabi, M. A.; Ashkhourian, J. T. Anal. Sci., 2012, 28, 1225-1230.

20. Russell, M.; Foreman, S. Cogent Chem., 2015, 1, 4-11. 